\title{
Nano-drug delivery systems in wound treatment and skin regeneration
}

\author{
Wei Wang ${ }^{1,2 \dagger}$, Kong-jun $\mathrm{Lu}^{2 \dagger}$, Chao-heng Yu ${ }^{3}$, Qiao-ling Huang ${ }^{1 *}$ and Yong-Zhong Du ${ }^{2^{*}}$
}

\begin{abstract}
Skin damages are defined as one of most common lesions people suffer from, some of wounds are notoriously difficult to eradicate such as chronic wounds and deep burns. Existing wound therapies have been proved to be inadequate and far from satisfactory. The cutting-edge nanotechnology offers an unprecedented opportunity to revolutionize and invent new therapies or boost the effectiveness of current medical treatments. In particular, the nanodrug delivery systems anchor bioactive molecules to applied area, sustain the drug release and explicitly enhance the therapeutic efficacies of drugs, thus making a fine figure in field relevant to skin regeneration. This review summarized and discussed the current nano-drug delivery systems holding pivotal potential for wound healing and skin regeneration, with a special emphasis on liposomes, polymeric nanoparticles, inorganic nanoparticles, lipid nanoparticles, nanofibrous structures and nanohydrogel.
\end{abstract}

Keywords: Nano-drug delivery system, Skin regeneration, Wound treatment

\section{Introduction}

Skin, the largest organ of human body, functions as a pivotal barrier featured with immunologic, sensorial and protective capability. Owing to its exposure to the external environment, skin is vulnerable to a variety of external factors which result in different types of skin damage and injury. It should be noticed that the prevalence of people suffering from chronic wounds has risen sharply in recent years, due to the dramatically increasing incidence of obesity and chronic diseases such as diabetes, venous and arterial insufficiency [1]. It is estimated that chronic wounds affect about $1-2 \%$ of the European and United states population [2], for example, prevalence of diabetes ulcers alone has already reached as high as $10-22 \%$ in diabetes patients [3]. However, traditional therapies generally involve costly and long-lasting treatments with a ulcer relapse rate of above 70\% [4]. The astonishing numbers of patients being eager for better healing quality and

*Correspondence: hql6512@163.com; duyongzhong@zju.edu.cn

tWei Wang and Kong-jun Lu contributed equally to this work

1 Department of Pharmaceutics, Hangzhou Third Hospital,

Hangzhou 310009, China

${ }^{2}$ Institute of Pharmaceutics, College of Pharmaceutical Sciences, Zhejiang

University, Hangzhou 310058, China

Full list of author information is available at the end of the article the staggering budget spent for wound care, which are still on the wax, prominently drive the research in fields of wound healing and skin regeneration.

Thanks to the innovative and impressive development of nanotechnology, numerous nano-drug delivery systems (nano-DDSs) were invented and introduced into the areas relevant to skin regeneration. It is universally testified that nano-DDSs evidently accelerate wound healing and improve the healing quality for the several prominent advantages they enjoy: (1) Nano-DDSs are found to be non-toxic, perfectly compatible with skin and favorably create a beneficial moist environment for activation and acceleration of wound healing process. (2) Some specific nano-DDSs are equipped with ability of entering into the cytoplasmic space across cellular barriers or activating specific transport mechanisms to improve the drug retention [5]. (3) When incorporated with bioactive molecules, nano-DDSs protect drugs from degradation elicited by proteases in wounds, remarkably enhancing therapeutic effectiveness [6]; (4) The sustained drug release also prolongs the maintenance of effective drug concentration, reduces the frequency of administration and leads to decline of cost as well as improvement of compliance. 
This review mainly introduces the wound healing process, the current wound treatment and their limitations, and the state of the art in nano-DDSs that holds a promising potential for future application, with a special focus on liposomes, polymeric nanoparticles, lipid nanoparticles, nanofibrous structures and nanohydrogel.

\section{Wound categories and wound healing process Wound categories}

Wounds are defined as the breakage or disruption of skin caused by trauma or medical/physiological conditions. Under such circumstance, damage to skin anatomical structure and the loss of skin physiological functions occur frequently. The wounds generally fall into two categories: acute wounds often result from mechanical damage or exposure to extreme heat, irradiation, electrical shock or corrosive chemicals. Such wounds heal in a relatively short period of time if supported by appropriate wound management [7]. Chronic wounds normally appear to be the complication of some specific diseases like diabetes, which is notorious for its horrendous incidence of ulcers. These wounds require for longer time to heal and their reoccurrence rates are extremely high unless the root diseases are cured [8].

According to wound depth, the wounds can be classified as three kinds: superficial wounds (only lose a part of epidermis), partial thickness wounds (epidermis and deeper dermal layers are affected) and full thickness wounds (subcutaneous fat and deeper tissue are disrupted) [9].

\section{Wound healing process}

Wound healing is a complex and dynamic physiological process involves with various cells, mediators, extracellular matrix (ECM) components, growth factors, and proteinases [10]. As showed in Fig. 1, it can be generally divided into three overlapping phases including inflammatory, proliferative, and re-epithelialization/remodeling phase $[11,12]$.

The inflammatory phase often lasts 2 to 5 days after skin damage. When an injury occurs, the hemostasis is initiated immediately by intravascular platelets to form a clot and stop bleeding [13]. Furthermore, platelets will be activated by thrombin and release several growth factors such as epidermal growth factor (EGF), insulin-like growth factor 1 (IGF-1), platelet-derived growth factor (PDGF), fibroblast growth factor (FGF), transforming growth factor (TGF- $\alpha$ and TGF- $\beta$ ) $[14,15]$. These growth factors diffuse into wound tissue and serve as biological signals to attract neutrophils, monocytes, leukocytes and macrophages, which will further mediate the inflammation, protect skin from infection and secret more growth factors to accelerate wound healing $[16,17]$.
The proliferative phase generally takes 3 days to 2 weeks after injury, featured with cell proliferation and migration [18]. Fostered by proangiogenic factors such as PDGF released by platelets and inflammatory cells within wound area, new blood vessels and capillaries gradually take shape [19]. Simultaneously with angiogenesis, migration of fibroblasts is also elicited by the stimulation of PDGF and FGF from inflammatory cells to form granulation tissue [20, 21]. With the accumulation and proliferation of fibroblasts, new ECM composed of collagen, proteoglycans, and elastin is produced. Some fibroblasts even differentiate into myofibroblasts and play a role in the contraction of wound area [22]. Moreover, activated keratinocytes around wound margin migrate to injured area to complete re-epithelialization [23].

Re-epithelialization and remodeling phase varies from 3 weeks to 2 years post-injury. The collagen III in newlysynthesized ECM is gradually replaced by collagen I and the new born collagen fibers evolve into a more organized lattice structure, augmenting tensile strength of healed skin $[24,25]$. Remodeling phase also concerns about scar formulation [26].

\section{Current wound treatment}

The ultimate goal of the wound management is to prevent serious infection, accelerate wound healing and reduced scars and pain for patients. Currently, a set of strategies are available for wound management mainly including debridement, autografts and application of therapeutic agents. In addition, some burgeoning new therapies such as stem cell therapy, gene therapy, photothermal and photodynamic therapy, are playing an increasingly vital role in some complicated wound treatment.

\section{Debridement}

Conventional debridement removes the necrotic or infected tissue that may prolong the inflammatory phase and impede wound contraction as well as re-epithelialization, fostering a better wound bed for healing process [27]. Debridement, including surgical, autolytic, mechanical, maggot, and enzymatic method, usually involves with further application of wound dressings [28]. Although debridement, especially the sharp debridement, has been well acknowledged as the gold standard for rapid removal of necrotic tissue and prevention of potential infection, it is still confronted with some limitations: its lasting and significant pain sometimes can be unacceptable for patients $[29,30]$, and it requires for experienced clinicians and specific materials in order to avoid second trauma. Therefore, applied method should be in accordance with the evaluation of wound characteristics, patient condition, and resources available in treatment [31]. 


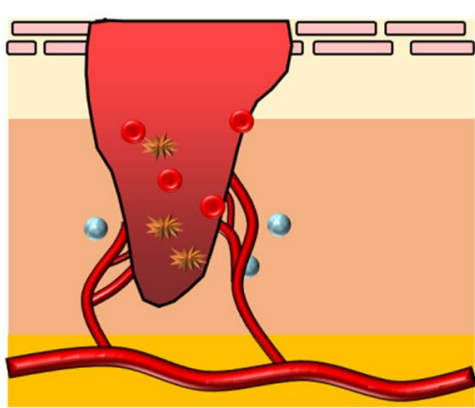

Trauma

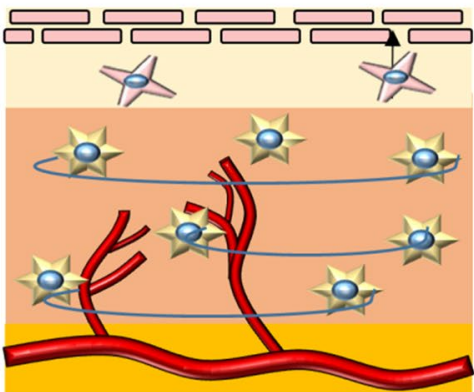

\section{Re-epithelialization}

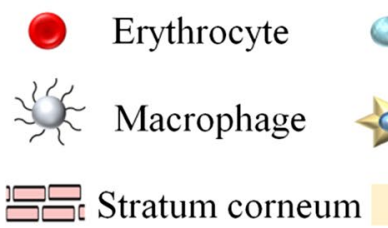

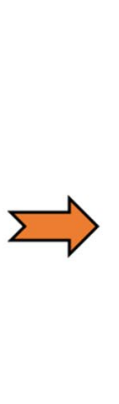

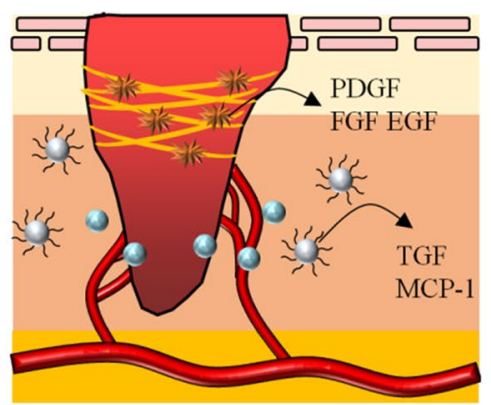

Inflammation
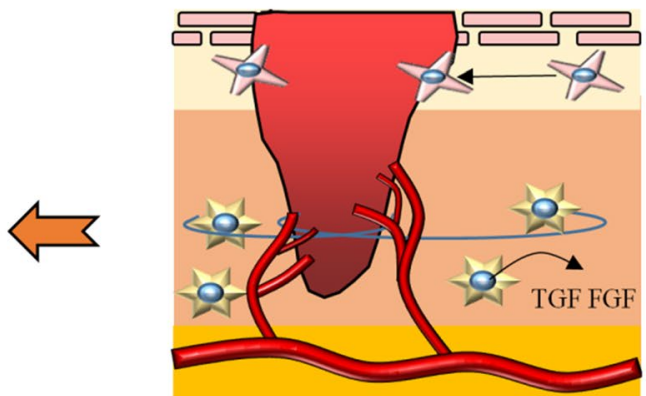

\section{Proliferation}

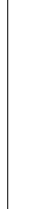

Fig. 1 Illustration of wound healing process

\section{Autografts and allografts}

The use of autografts and allografts remains the gold standard for skin regeneration. Autografts and allografts approaches mainly harvest full-thickness fascia from a donor site of patients or other donators and graft it over the target region [32]. Autografts have reputation for excellent adhesion to the wound site and better cosmetic results, conspicuously relieving pain and reducing rejection. However, the rigorous requirement of donor site limits their usage and such skin grafts also bring the undesirable amount of scar and serious skin contraction in late stage of wound healing, along with the costly hospital stay [33]. As for allografts, the major advantage is the temporary prevention of wound dehydration and contamination, along with the favorable fitness to wound. Nevertheless, due to the resource of grafts, the risk of disease transmission and higher rate of immune rejection are inevitable [34]. It should be noticed that the tissueengineered skin substitutes and in situ biofabrication of skin substitutes like cultured epithelial autografts, are emerging as promising strategies to overcome the setbacks of traditional autografts [35].

\section{Topical drug application}

Application of topical drugs, which mainly focuses on promoting healing process and preventing infection, still plays an indispensable role in treatment for all types of wounds. Hence, a large demand still exists in exploring novel therapeutic agents for topical wound therapy. Topical therapeutic agents consist of growth factors and antimicrobial agents being crucial for the wound treatment and skin regeneration. 
Growth factors are biologically active polypeptides which regulate cell growth, differentiation, and migration and exert an impact on all stage of wound healing. It has been confirmed in some clinical researches, growth factors exert amazing effects on wound healing promotion and skin function restoration without any obvious side effects. The clinical used growth factors are listed in Table 1.

Owing to infection being a leading cause of mortality and horribly delaying the wound healing, antimicrobial agents are generally administrated both topically and systemically. The choice of antimicrobial agents strongly depends on the microbiological analysis for species and susceptibility of microorganisms. The most commonly used antimicrobial agents are listed in Table 2.

\section{Nano-drug delivery system in wound treatment and skin regeneration}

Nano-DDSs hold immense potential in enhancement of drug therapeutic efficacy for their capability of preventing drug degradation and sustaining drug release. Numerous nano-DDSs carrying therapeutic agents are springing up unprecedentedly and adopted in promoting wound healing and skin regeneration, mainly including liposomes, polymeric nanoparticles, inorganic nanoparticles, lipid nanoparticles, nanofibrous structures and nanohydrogel [36-38] (Fig. 2). Recent researches of nano-DDSs are listed in Table 3.

\section{Liposomes}

Liposomes are bilayer vesicles built by amphiphilic molecules such as phospholipids, emerging as one of promising nano-carriers for topical drug delivery [39]. They are nontoxic, biodegradable, biocompatible with skin, and capable of accommodating both hydrophilic

Table 1 Growth factors in clinical application

\begin{tabular}{|c|c|c|c|c|}
\hline Growth factor & Target cells & Administration & Function & Refs. \\
\hline EGF & $\begin{array}{l}\text { Fibroblasts } \\
\text { Keratinocytes }\end{array}$ & Topical & $\begin{array}{l}\text { Promote cell proliferation, differentiation and migration; accelerate epidermal } \\
\text { regeneration }\end{array}$ & [93] \\
\hline PDGF & $\begin{array}{l}\text { Neutrophils } \\
\text { Macrophages } \\
\text { Fibroblasts } \\
\text { Smooth muscle cells }\end{array}$ & Topical & $\begin{array}{l}\text { Increase the structural integrity of vessels; promote cell proliferation, ECM Depo- } \\
\text { sition and re-epithelialization }\end{array}$ & [94] \\
\hline bFGF & $\begin{array}{l}\text { Keratinocytes } \\
\text { Fibroblasts }\end{array}$ & Topical & Promote collagenase production, ECM deposition and re-epithelialization & {$[95]$} \\
\hline GM-CSF & $\begin{array}{l}\text { Keratinocyte } \\
\text { Endothelial cells } \\
\text { Macrophages Eosinophils }\end{array}$ & $\begin{array}{l}\text { Topical/sub- } \\
\text { cutaneous } \\
\text { injection }\end{array}$ & $\begin{array}{l}\text { Promote local recruitment of inflammatory cells, stimulate cell proliferation and } \\
\text { differentiation and wound contraction }\end{array}$ & {$[96]$} \\
\hline TGF- $\beta$ & $\begin{array}{l}\text { Keratinocytes } \\
\text { Macrophages } \\
\text { Lymphocytes } \\
\text { Fibroblasts }\end{array}$ & Topical & $\begin{array}{l}\text { Promote granulation tissue formation; re-epithelialization; matrix formation and } \\
\text { remodeling }\end{array}$ & [97] \\
\hline
\end{tabular}

Table 2 Most commonly used antimicrobial agents in wound treatment

\begin{tabular}{|c|c|c|c|}
\hline Antimicrobial agents & Administration & Spectrum & References \\
\hline Gentamicin & Systemic/topical & Gram-positive bacteria & [98] \\
\hline Tetracycline & Oral/topical & Gram-positive and Gram-negative bacteria & {$[99,100]$} \\
\hline Ciprofloxacin & Oral/systemic & Gram-positive and Gram-negative bacteria especially Gram-negative bacilli & {$[101,102]$} \\
\hline Vancomycin & Systemic & Gram-positive bacteria especially MRSA & {$[103]$} \\
\hline Penicillin G & Systemic & Non- $\beta$-lactamase-producing Gram-positive bacteria, anaerobes & {$[104]$} \\
\hline Neomycin & Systemic/topical & Aerobic Gram-negative bacilli and Gram-positive aerobes & {$[105]$} \\
\hline Polymyxin B & Systemic & Gram-negative bacteria & {$[106]$} \\
\hline Mupirocin & Topical & Gram-positive bacteria especially MRSA, some Gram-negative flora & {$[107]$} \\
\hline Amphotericin B & Systemic/topical & Fungi & {$[108]$} \\
\hline Silver sulfadiazine & Topical & Gram-positive, most Gram-negative bacteria, and some fungal forms & {$[109]$} \\
\hline Mafenide acetate & Topical & Gram-negative bacilli, anaerobes & [110] \\
\hline
\end{tabular}



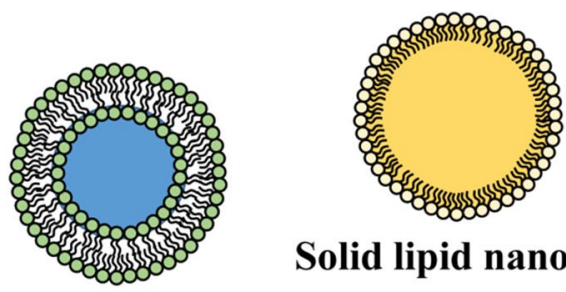

Solid lipid nanoparticles
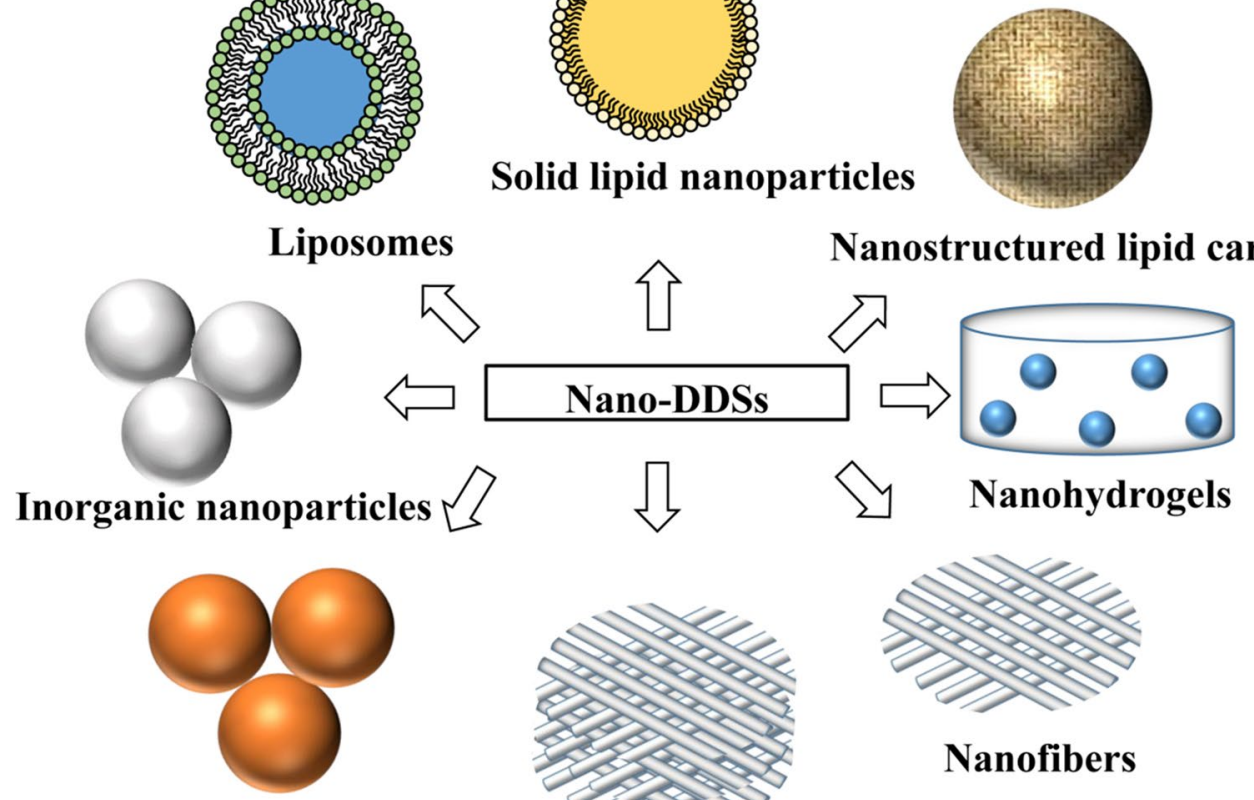

Polymeric nanoparticles
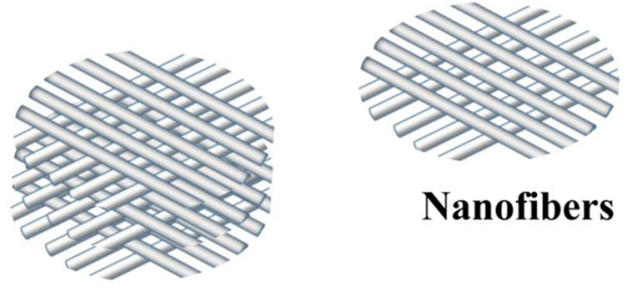

Nanoscaffolds

Fig. 2 Nano-drug delivery systems in skin regeneration and wound treatment

drugs (e.g. growth factors) in inner water cavity and hydrophobic agent in bilayer [40, 41]. In this way, liposomes provide protection for encapsulated drug and sustain the drug release. Furthermore, liposomes effectively cover wound and create moist environment on wound surface after application, which is very conducive to wound healing [42]. Taking advantage of all these merits, liposomes have been universally applied in wound treatment and skin regeneration. Xu et al. [43] prepared a novel liposome with hydrogel core of silk fibroin which effectively encapsulated bFGF. The vehicles remarkably improved the stability of bFGF in wound fluids and maintained cell proliferation activity with respect to traditional liposomes. Furthermore, the liposomes with hydrogel core efficiently accelerated wound healing, particularly in inducing angiogenesis. Nunes et al. [44] evaluated the promoting effect of a gelatin-based membrane containing usnic acid-loaded liposome on wound healing. The experiments on a porcine model indicated that the liposomal membrane conspicuously controlled the secondary infection. In addition, more exuberant and cellularized granulation tissue with better collagen deposition was observed in the liposomal membrane treated group, therefore the special membrane boasted a comparable capacity to commercial product DuoDerme with regard to enhance maturation of granulation tissue and scar repair.

Presented as a new generation of liposomes, deformable liposomes, also called transfersomes, mainly consist of phospholipids and an edge activator (such as sodium cholate, sodium deoxycholate and Tween-80) [45], bringing new insight into topical drug delivery. These novel carriers not only integrate the benefits of traditional liposomes, but show more merits in topical application. The presence of edge activator renders high flexibility of deformable liposomes and enables them to across the stratum corneum and reach the viable epidermis [46]. Uk Choi et al. [47] conjugated low-molecular-weight protamine (LMWP) to the N-termini of EGF, PDGF-A and IGF-1, these molecules were subsequently complexed with hyaluronic acid and eventually incorporated into cationic deformable liposomes. The results showed that the cationic elastic liposomes containing the growth factor complex significantly accelerated the wound closure rate in the diabetic mouse model, with the maximal shrink of wound size by $58 \%$ compared with the native growth factor complex. It was fully confirmed that the elastic liposomes cooperated with growth factor complex, synergistically exerting both rapid and prolonged effects on promoting chronic wound healing. A new 


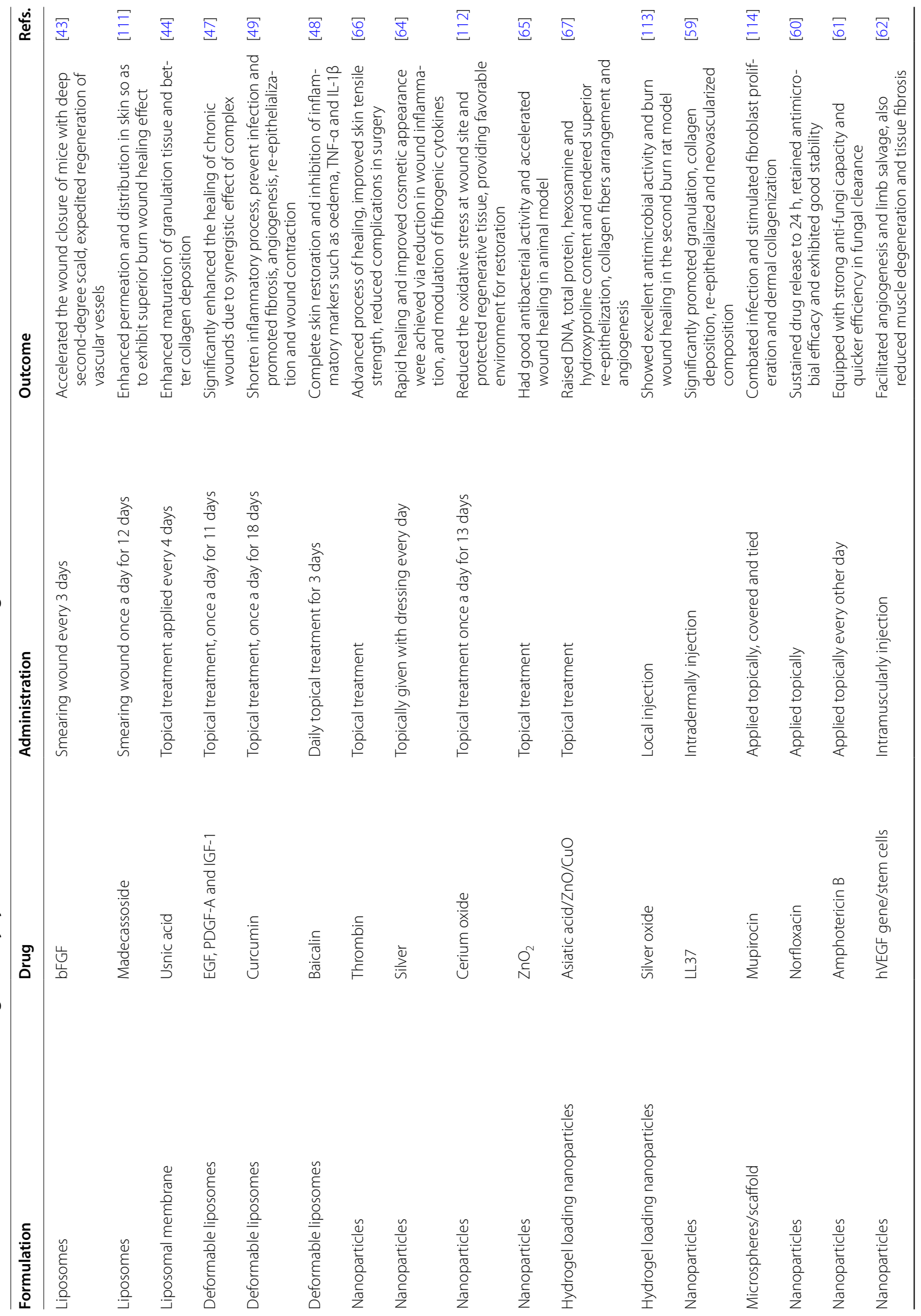




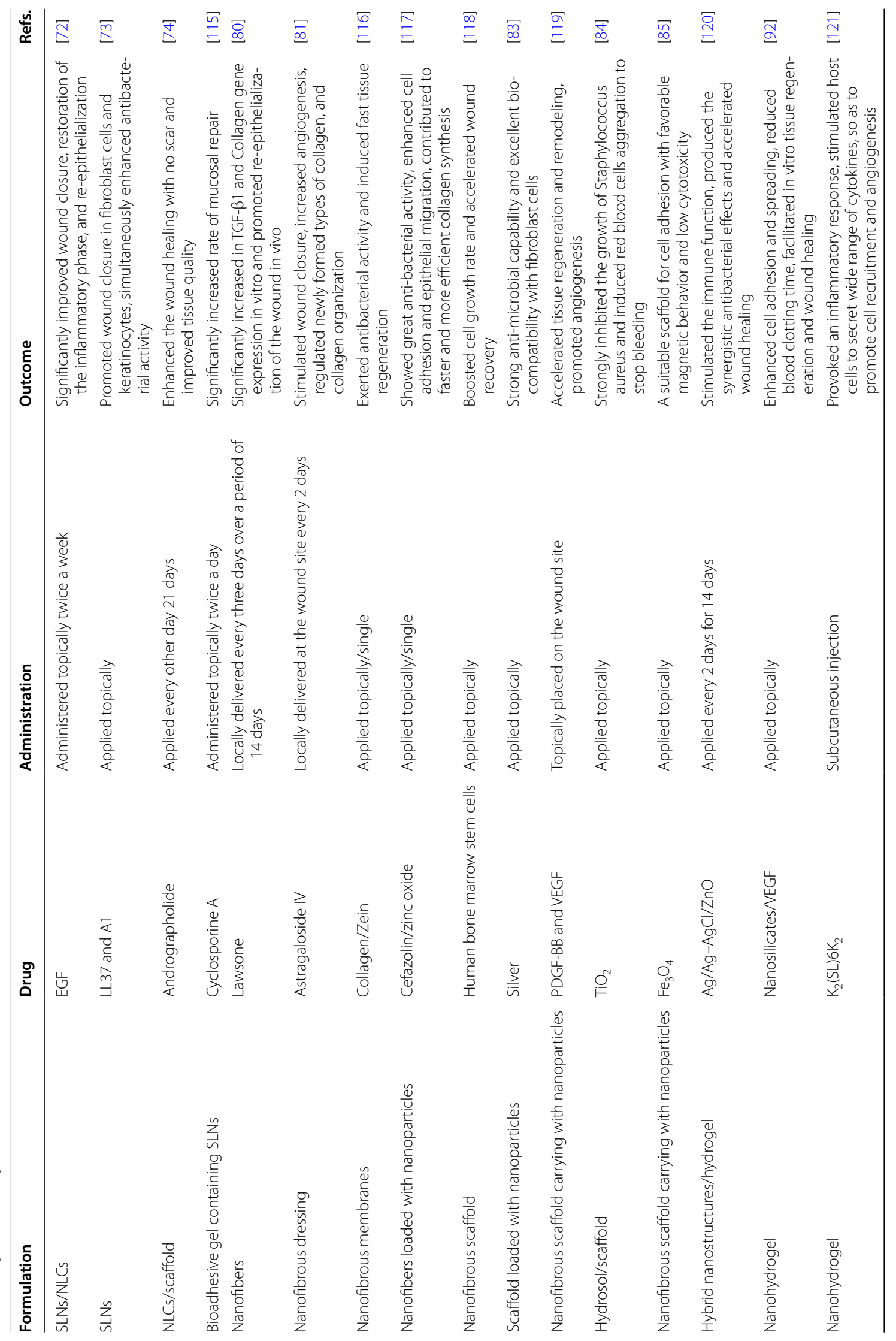




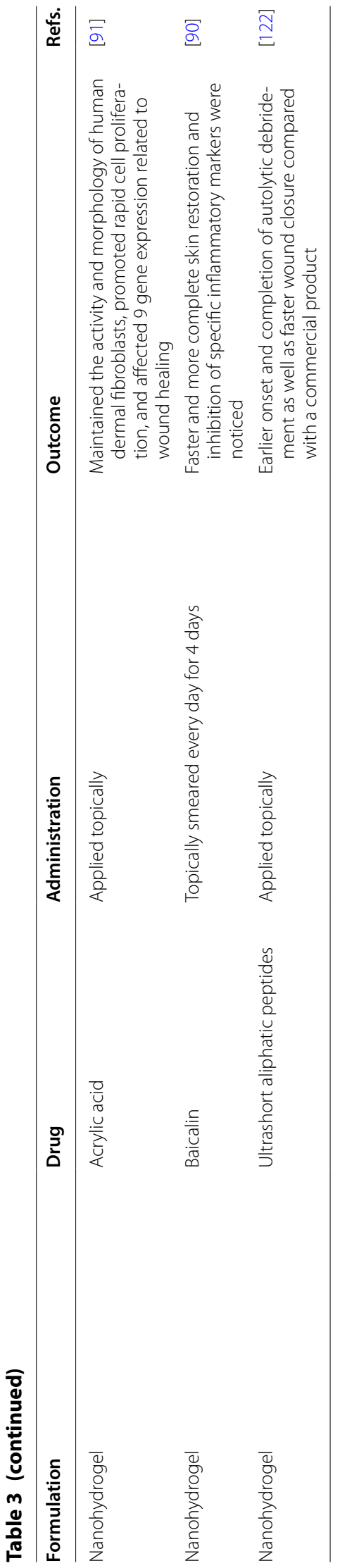


self-assembling core-shell gellan-transfersome loading baicalin was designed by Manconi et al. [48], they found out that novel transfersomes showed a relatively high skin drug deposition, about $11 \%$ of baicalin was retained in the whole skin, $8 \%$ of which was in the dermis, considered to be quite efficient. Daily application of baicalin transfersomes in mice model brought about complete skin restoration and inhibition of inflammatory markers such as oedema, TNF- $\alpha$ and IL-1 $\beta$. Kianvash et al. [49] also noticed that their newly prepared propylene glycol nanoliposomes containing curcumin not only featured by preferential physiochemical properties (small size, sustained drug release, good stability and biocompatibility), but promoted second degree burns in rat model in terms of avoiding infections and elevating wound contraction.

Nevertheless, liposomes also exhibit some demerits in application: drug leakage in liposomes sometimes can be unavoidable and rapid [50, 51]; the low reproducibility and stability of liposomes remains a major obstacle for its expansion in clinical use $[52,53]$.

\section{Polymeric nanoparticles}

Polymeric nanoparticles are biocompatible colloidal systems drawing increasing attention in both biomedicine and bioengineering fields [54]. When embedded or conjugated with these polymeric devices, drugs are protected from degradation by the proteases presenting in the wound and released in a controlled manner so as to reduce administration frequency. The need of effectively delivering biomolecules such as antimicrobial agents, growth factors and genes, will be met with aid of nanoparticles $[55,56]$. Currently most polymeric nanoparticles are prepared by poly lactic-co-glycolic acid (PLGA, crowned as the mostly used polymers), alginate, gelatine, chitosan, as well as other polymer combinations $[57,58]$.

Many researches focus on developing polymeric nanoparticles encapsulating antimicrobial agents. Chereddy et al. [59] reported a PLGA nanoparticle loaded with antimicrobial peptide LL37 (PLGA-LL37 NPs) could be a biodegradable drug delivery system that accelerated healing process. It displayed antimicrobial activity on Escherichia coli and induced promoted cell migration while lifting no effect on proliferation of keratinocytes. In full thickness excisional wound model, PLGA-LL37 NPtreated group exhibited advanced granulation tissue formation, characterized by significant higher collagen deposition, re-epithelialized composition and neovascularization. Furthermore, it improved angiogenesis and modulated the inflammatory wound response by up-regulation of interleukin-6 (IL-6) and vascular endothelial growth factor (VEGF). Dave et al. [60] prepared a lipidpolymer hybrid nanoparticle formulation which was able to sustained drug release to $24 \mathrm{~h}$ with favorable skin permeation and reduced the frequency of application. Norfloxacin -loaded nanoparticles still performed well in antimicrobial efficacy test against Staphylococcus aureus and Pseudomonas aeruginosa. Therefore, it was considered to hold a broad prospect in treating burn-induced infections.

To reduce high cytotoxicity of Amphotericin B and improve the patient appliance, Sanchez et al. [61] incorporated Amphotericin B into a silane-based hydrogel nanoparticles to replace the traditional intravenous injection infusion. Amphotericin B nanoparticles resulted in equivalent or enhanced killing efficacy with $72.4-91.1 \%$ by $4 \mathrm{~h}$ for Clinical strains. It also contributed to statistically significant reduction of fungal biofilm metabolic activity ranging from 80 to $95 \%$. In a murine full-thickness burn model, Amphotericin B nanoparticles cleared fungi in a more rapid manner versus free drug solution within 3 days while their wound healing rates were similar.

Performance of nanoparticles also exceeds expectation in gene therapy related to skin regeneration. To overcome the setbacks of insufficient expression of angiogenic factors and low cell viability after transplantation, biodegradable nanoparticles were developed to deliver hVEGF gene to human mesenchymal stem cells and human embryonic stem cell-derived cells [62]. hVEGF production, cell viability, and engraftment into target tissues of stem cells were prominently enhanced. The scaffold seeded with genetically modified stem cells directly achieved 2-4 fold higher vessel densities 2 weeks postimplantation versus control cells or cells transfected with hVEGF gene by Lipofectamine 2000. When intramuscularly injected into mouse ischemic hindlimbs, cells pretreated with nanoparticles still facilitated angiogenesis and limb salvage, meanwhile, reducing muscle degeneration and tissue fibrosis.

\section{Inorganic nanoparticles}

Inorganic nanoparticles refer to nanoparticles deprived from inorganic materials, including the metallic nanoparticles, carbon based nanoparticles, ceramic nanoparticles etc. [63]. Benefiting from its intrinsic nature of materials, inorganic nanoparticles exhibit both the similar merits in wound healing treatment and strong antibacterial effect, for example, silver nanoparticles are often applied as antimicrobial agents. Therefore, the combination of inorganic nanoparticles is more preferred in research to achieve synergistic promoting effect of both materials and drugs.

Jun et al. [64] investigated the promoting woundhealing effect of silver nanoparticles and its mechanism were systematically revealed in burn wound and chronic wound model. Dose-dependent rapid healing and 
improved superficial wound appearance were observed and further studies demonstrated that the relatively prompt wound healing and reduced wound inflammation may be mediated by elevation of TGF- $\beta$, VEGF, IL- 6 induced by silver nanoparticles.

Ali et al. [65] designed and synthesized $\mathrm{ZnO}_{2}$ nanoparticles by co-precipitation method. This kind of nanoparticles is an efficient inorganic material with antibacterial properties. $\mathrm{ZnO}_{2}$ nanoparticles had good antibacterial activity for pseudomonas aeruginosa and aspergillus isolated from wound infected tissues of burn patients. The results of histopathological evaluation confirmed that $\mathrm{ZnO}_{2}$ nanoparticles could accelerate the healing of skin wounds in animal models in vivo.

Thrombin is a widely used drug for topical hemostasis and wound healing. With the aim of overcoming the weak stability of drug, a thrombin-bounded maghemite $\left(\mathrm{Fe}_{2} \mathrm{O}_{3}\right)$ nanoparticle were fabricated and its therapeutic effect were evaluated via application on an incisional wound model [66]. The results illustrated that the nanoparticle-treated group characterized by fewest inflammatory cells, smallest amount of granulation tissue along the surgical scar and highest values of skin tensile strength. All the evidences supported that thrombin-bounded maghemite nanoparticle remarkably advanced the wound healing stage and achieved better healing quality.

Studies have been extended to the blending application of different inorganic nanoparticles to render a better efficacy. Thanusha et al. [67] developed a hydrogel co-encapsulated with asiatic acid and nanoparticles (zinc oxide and copper oxide) for second burn wound healing. Physicochemical studies showed the formulation was characterized by porous morphology, large water uptake, excellent tensile strength and good anti-bacterial capacity. Thanks to the co-loaded nanoparticles, in vivo study of therapeutic efficiency demonstrated that DNA, total protein, hexosamine and hydroxyproline content of wounds in hydrogel treated group were all raised significantly; re-epithelialization, collagen fibers arrangement and angiogenesis were confirmed to be more superior than the group treated with commercial available products.

\section{Lipid nanoparticles}

Solid lipid nanoparticles (SLNs) and nanostructured lipid carriers (NLCs) were representatives of lipid nanoparticles introduced to overcome the limitation of liposomes. Lipid nanoparticles were generally prepared with physiological lipids or lipid molecules and their preparation process requires no involvement of any potentially toxic organic solvents [68]. Their nontoxic colloidal dimensions contribute to the controlled release of drug and versatility of administration $[69,70]$. The potential of lipid nanoparticles for topical therapeutic or cosmetic purposes has been partially exploited as a market product loaded with Q10 is available (NanoRepair Q10 ${ }^{\circledR}$, Dr. Rimpler) [71].

Gainza et al. [72] reported both SLNs and NLCs loading with rh-EGF for treatment of chronic wounds. Both of the nano-formulations were prepared through emulsification-ultrasonication method, but the preparation process of NLCs involved no organic solvent and characterized by much higher encapsulation efficiency. The results of the effectiveness of nano-formulations showed that both of them equipped with superior capability on promoting cell proliferation compared with free rh-EGF, and significantly improved healing in terms of wound closure, restoration of the inflammatory process, and reepithelialization when applied on full-thickness wound model in $\mathrm{db} / \mathrm{db}$ mice.

Fumakia et al. [73] fabricated SLNs loading with an elastase inhibitor serpin A1 and antimicrobial peptide LL37 to achieve synergistic effect on wound healing. Making the most of the synergistic effect of drugs and extraordinary characteristics of SLNs, the formulation promoted wound closure in fibroblast cells and keratinocytes. In addition, it simultaneously enhanced antibacterial activity against $S$. aureus and $E$. coli compared with the group treated with LL37 or A1 alone.

In another related study, andrographolide-loaded lipid nanoparticles were developed, optimized, then successfully incorporated into a chitosan-HA scaffold [74]. This scaffold exhibited appropriate porosity, suitable swelling ratio and a controlled drug release behavior up to $72 \mathrm{~h}$. When applied to second degree burn wounds, it notably reduced scar formation and improved healing quality, which could be explained by anti-inflammatory and antioxidant effect of chitosan, HA and nanoparticles. Therefore, this scaffold would be a potential candidate for wound healing treatment.

\section{Nanofibrous structures}

Nanofibers are fabricated from natural and synthetic continuous polymer chains which are able to subsequently act as nanofibrous sheets or 3D-scaffolds applied in tissue engineering $[75,76]$. These nanofibrous structures are designed to mimic the ECM, provide favorable condition for cell attachment and elevate cell-drug interaction, serving as a replacement for artificial dermal analogues [76, 77]. Electrospinning is the most widelyadopted technique for production of nanofibers. An electrical charge is taken as driving force to draw fibers from a polymer solution so as to fabricate nanometric continuous fibers [78]. Due to its high area to volume ratio, the nanofibers enhance transfer of a variety of therapeutic 
agents including diverse antimicrobial agents, growth factors and even nucleic acids [79].

Adeli-Sardou et al. [80] electrospun lawsone into polycaprolactone-gelatin $(\mathrm{PCL} / \mathrm{Gel})$ polymers in core-shell architecture, so as to produce special nanofibers for skin tissue regeneration. In addition to boosted cell attachment and proliferation brought along by nanofibers, results of RT-qPCR revealed that in vitro gene expression of TGF- $\beta 1$, collagen and EGF was impressively elevated in the nanofiber-treated cells. Furthermore, $1 \%$ lawsone $\mathrm{PCL} / \mathrm{Gel}$ had the best impact on wound healing of rats, especially in the acceleration of re-epithelialization. Shan et al. [81] prepared astragaloside IV loaded silk fibroin/ gelatin nanofibrous dressing via electrospinning nanotechnology. The nanofibrous dressing was equipped with excellent biocompatibility, significantly improving cell adhesion and proliferation in vitro, accelerating wound healing and inhibiting scar formation in vivo. Results also found out that the nanofibrous dressing exerted positive impacts on angiogenesis, collagen production and collagen organization.

Combined with stem cell therapy, Ramasatyaveni et al. [82] attached mouse bone marrow stem cells to a porous polyethylene glycol-polyurethane (PEG-PU) scaffold to better fulfill differentiation potential and wound healing capability of stem cells. The results of in vivo observations depicted significant increase in fibroblast proliferation, collagen deposition and anti-oxidant enzyme activity, with obvious decreased expression of pro-inflammatory cytokines (IL-1 $\beta$, TNF- $\alpha$, IL-8, etc.) and concomitant increase in anti-inflammatory cytokines (IL-10, IL-13) at an early healing stage. Furthermore, enhanced engraftment and vascularity were detected to provide evidences for an accelerated wound tissue closure.

In some cases, nanofibrous structures were simultaneously integrated with other nano-formulations to achieve a synergic impressive effect on skin regeneration. Zulkifli et al. [83] fabricated the hydroxyethyl cellulose-silver nanoparticles into scaffold to endow it with anti-microbial capability. Fibroblast cells were able to adhere onto the surface of scaffolds after co-incubation, indicating that it is a potential substrate with high biocompatibility for biomedical applications, especially in the wound healing and tissue engineering field. According to report of Fan et al. [84], a nanofibrous scaffold carrying with nano$\mathrm{TiO}_{2}$ hydrosol was designed for better skin repair. The results of physicochemical properties revealed the good permeability and stability, which offer a humid environment for wound healing and met the requirement of wound coverage protection. Due to the embedment of nano- $\mathrm{TiO}_{2}$, the scaffold strongly inhibited the growth of Staphylococcus aureus and induced red blood cell aggregation to stop bleeding. In another study, magnetic iron oxide nanoparticles were incorporated into three-dimensional fibrous scaffolds to form a novel formulation and its physicochemical properties and cell biocompatibility in vitro were investigated [85]. It turned out that magnetic iron oxide nanoparticles were successfully loaded into scaffolds while maintaining magnetic behavior. It was also a suitable scaffold for cell adhesion with low cytotoxicity, thus having prominent advantages in skin tissue engineering, particularly in the treatment that may encounter magnetic field.

\section{Nanohydrogel}

Nanohydrogel is the three-dimensional polymeric networks considered as ideal formulation for wound management: the porous three-dimensional structure endows it with the ability of aqueous fluid absorption [86], preventing wound dehydration and creating a beneficial moist environment for wound healing [87]; its nonadhesive nature allows it to preserve the wound bed while maintaining the penetration of oxygen, which is necessary for wound healing [88]; soft texture of nanohydrogel provides comfortable experience in the course of treatment [89]. Furthermore, nanohydrogel is able to encapsulate many related drugs with perfect compatibility and efficacy, exerting an impressive effect on skin regeneration.

A gellan-cholesterol nanohydrogel embedding baicalin was introduced to speed up wound healing process [90]. Characterized by proper viscosity, improved skin retention and preferable biocompatibility, it was further applied to a cutaneous inflammation mice model induced by a phorbol ester. The baicalin-loaded nanohydrogel exhibited optimal performance for a complete skin restoration and inhibition of specific inflammatory markers (i.e., myeloperoxidase, tumor necrosis factor- $\alpha$ and oedema) were realized in vivo. Xi Loh et al. [91] found that a newly-produced bacterial nanocrystal cellulose/ acrylic acid hydrogel could rapidly adhere to fibroblasts, maintain the activity and morphology of human dermal fibroblasts, limit cell migration, promote rapid cell proliferation, and affect 9 gene expression related to wound healing like IL-6, IL-10, GM-CSF, TGF- $\beta$ and matrix metalloproteinase-2 (MMP-2). Thus this hydrogel was regarded as playing a pivotal role in skin regeneration.

Besides, the versatile administration of nanohydrogel has received considerable interest, with the special focus on injectable nanohydrogel. Giriraj et al. [92] reported a nanocomposite hydrogel consisted of natural polysaccharide, $k$-carrageenan and nanosilicates. This specially designed nanohydrogel was confirmed to feature with high mechanical stiffness and good porosity with an interconnected network. With the addition of VEGF, VEGF-loaded nanohydrogel significantly enhanced cell 
adhesion and spreading, reduced blood clotting time and facilitated in vitro tissue regeneration. However, the further investigation in vivo is required to fully reveal the therapeutic efficacy of this novel formulation on wound healing.

\section{Conclusion}

The treatment of chronic wounds or ulcers remains a thorny and daunting challenge because current therapies mostly failed to provide favorable outcomes of wound healing. Nevertheless, the progressive expansion of nano-DDSs in recent years has brought new insight for skin regeneration of wounds: these drug carriers prolong drug release, protect drug from degradation and improve skin retention, so as to realize augment of the therapeutic power of biological and synthetic molecules (e.g. reduction or eradication of the wound bacterial load and improvement of re-epithelialization). Moreover, various combinations of nano-DDSs are served as synergistic platforms for delivery, some of which even mimic and offer perfect physiological environment for the healing process. Despite the enormous potential of nano-DDSs, these systems also have exposed some limitations in researches such as lack of international standards and evaluation methods on their toxicology, biocompatibility and targeting efficiency, as well as the undeniable restriction of industrial production for their complicated preparation procedures. However, it is an inevitable and unstoppable trend for researchers to further exploit the full potential of nano-DDSs, overcome the technical difficulties and bring tangible benefits for the patients suffered from wounds, nano-DDSs are bound to constitute the most promising and cost-effective therapies to boost the wound healing and skin regeneration.

\begin{abstract}
Abbreviations
nano-DDSs: nano-drug delivery systems; ECM: extracellular matrix; EGF: epidermal growth factor; IGF-1: insulin-like growth factor 1; PDGF: plateletderived growth factor; FGF: fibroblast growth factor; TGF: transforming growth factor; PLGA: poly lactic-co-glycolic acid; IL-6: interleukin-6; VEGF: vascular endothelial growth factor; SLNs: solid lipid nanoparticles; NLCs: nanostructured lipid carriers; GM-CSF: granulocyte-macrophage colony-stimulating factor; MMP: matrix metalloproteinase.
\end{abstract}

\section{Acknowledgements}

Not applicable.

\section{Authors' contributions}

YD brought forward the subject and guided the writing, WW and KL were major contributors in writing the manuscript. All authors read and approved the final manuscript.

\section{Funding}

This study was supported by Hangzhou Science and Technology Development Plan (20191203B114) and the New Century 151 Talent Project of

Zhejiang Province.
Availability of data and materials

Not applicable.

Ethics approval and consent to participate

Not applicable.

\section{Consent for publication}

Not applicable.

\section{Competing interests}

The authors declare that they have no competing interests.

\section{Author details}

${ }^{1}$ Department of Pharmaceutics, Hangzhou Third Hospital, Hangzhou 310009, China. ${ }^{2}$ Institute of Pharmaceutics, College of Pharmaceutical Sciences, Zhejiang University, Hangzhou 310058, China. ${ }^{3}$ Department of Burn, Second Affiliated Hospital, School of Medicine, Zhejiang University, Hangzhou 310009, China.

Received: 25 March 2019 Accepted: 28 June 2019

Published online: 10 July 2019

\section{References}

1. Kuehn BM. Chronic wound care guidelines issued. JAMA. 2007;297:938.

2. Schreml S, Szeimies RM, Prantl L, Landthaler M, Babilas P. Wound healing in the 21st century. J Am Acad Dermatol. 2010;63:866-81.

3. Margolis DJ, Hoffstad O, Nafash J, Leonard CE, Freeman CP, Hennessy S, Wiebe DJ. Location, location, location: geographic clustering of lowerextremity amputation among medicare beneficiaries with diabetes. Diabetes Care. 2011;34:2363.

4. Garcia-Orue I, Gainza G, Villullas S, Pedraz JL, Hernandez RM, Igartua M. Nanotechnology approaches for skin wound regeneration using drugdelivery systems. 2016. p. 31-55.

5. Sandhiya S, Dkhar SA, Surendiran A. Emerging trends of nanomedicine: an overview. Fundam Clin Pharmacol. 2010;23:263-9.

6. Losi P, Briganti E, Magera A, Spiller D, Ristori C, Battolla B, Balderi M, Kull S, Balbarini A, Di SR. Tissue response to poly(ether)urethane-polydimethylsiloxane-fibrin composite scaffolds for controlled delivery of proangiogenic growth factors. Biomaterials. 2010;31:5336-44.

7. Whitney JAD. Overview: acute and chronic wounds. Nurs Clin North Am. 2005;40:191-205.

8. Upton D, Solowiej K, Hender C, Woodyatt KY. Stress and pain associated with dressing change in patients with chronic wounds. J Wound Care. 2012;21:53.

9. Caló E, Khutoryanskiy W. Biomedical applications of hydrogels: a review of patents and commercial products. Eur Polymer J. 2015;65:252-67.

10. Han G, Ceilley R. Chronic wound healing: a review of current management and treatments. Adv Ther. 2017;34:1-12.

11. Madhumathi K, Sudheesh Kumar PT, Abhilash S, Sreeja V, Tamura H, Manzoor K, Nair SV, Jayakumar R. Development of novel chitin/nanosilver composite scaffolds for wound dressing applications. J Mater Sci Mater Med. 2010;21:807-13.

12. Moura LI, Dias AM, Carvalho E, de Sousa HC. Recent advances on the development of wound dressings for diabetic foot ulcer treatment-a review. Acta Biomater. 2013:9:7093-114.

13. Martin P. Wound healing-aiming for perfect skin regeneration. Science. 1997;276:75-81.

14. Braund R, Hook S, Medlicott NJ. The role of topical growth factors in chronic wounds. Curr Drug Deliv. 2007;4:195-204.

15. Gainza G, Villullas S, Pedraz JL, Hernandez RM, Igartua M. Advances in drug delivery systems (DDSs) to release growth factors for wound healing and skin regeneration. Nanomed Nanotechnol Biol Med. 2015;11:1551-73.

16. Kiritsy CP, Lynch AB, Lynch SE. Role of growth factors in cutaneous wound healing: a review. Crit Rev Oral Biol Med. 1993;4:729.

17. Eming SA, Krieg T, Davidson JM. Inflammation in wound repair: molecular and cellular mechanisms. J Invest Dermatol. 2007;127:514-25. 
18. Singer AJ, Clark RA. Cutaneous wound healing. N Engl J Med. 2007;341:738-46.

19. Velnar T, Bailey T, Smrkolj V. The wound healing process: an overview of the cellular and molecular mechanisms. J Int Med Res. 2009:37:1528-42.

20. Malinda KM, Sidhu GS, Banaudha KK, Gaddipati JP, Maheshwari RK, Goldstein AL, Kleinman HK. Thymosin a1 stimulates endothelial cell migration, angiogenesis, and wound healing. J Immunol. 1998;160:1001-6.

21. Tettamanti G, Grimaldi A, Rinaldi L, Arnaboldi F, Congiu T, Valvassori R, De EM. The multifunctional role of fibroblasts during wound healing in Hirudo medicinalis (Annelida, Hirudinea). Biol Cell. 2004;96:443-55.

22. Li B, Wang JH. Fibroblasts and myofibroblasts in wound healing: force generation and measurement. J Tissue Viability. 2011;20:108.

23. Carmen MM, Pratap G, Michael L, Joanne S, Jamie L, Diana N, Joan R, Min L, Jiang CK, Rochelle H. Wound healing is accelerated by agonists of adenosine A2 (Gas-linked) receptors. J Exp Med. 1997;186:1615-20.

24. Ehrlich HP, Keefer KA, Myers RL, Passaniti A. Vanadate and the absence of myofibroblasts in wound contraction. Arch Surg. 1999;134:494-501.

25. Stadelmann WK, Digenis AG, Tobin GR. Physiology and healing dynamics of chronic cutaneous wounds. Am J Surg. 1998;176:26S-38S.

26. Rai NK, Tripathi K, Sharma D, Shukla VK. Apoptosis: a basic physiologic process in wound healing. Int I Low Extrem Wounds. 2005;4:138.

27. Nusbaum AG, Gil J, Rippy MK, Warne B, Valdes J, Claro A, Davis SC. Effective method to remove wound bacteria: comparison of various debridement modalities in an in vivo porcine model. J Surg Res. 2012;176:701-7.

28. Kirshen C, Woo K, Ayello EA, Sibbald RG. Debridement: a vital component of wound bed preparation. Adv Skin Wound Care. 2006;19:506.

29. Kammerlander G, Andriessen A, Asmussen P, Brunner U, Eberlein T. Role of the wet-to-dry phase of cleansing in preparing the chronic wound bed for dressing application. J Wound Care. 2005;14:349.

30. Bradley M, Cullum N, Sheldon T. The debridement of chronic wounds: a systematic review. Health Technol Assess. 1999:3:1-78.

31. Steenvoorde P, Jacobi CE, Van DL, Oskam J. Maggot debridement therapy of infected ulcers: patient and wound factors influencing outcome - a study on 101 patients with 117 wounds. Ann R Coll Surg Engl. 2007:89:596-602.

32. Dreifke MB, Jayasuriya AA, Jayasuriya AC. Current wound healing procedures and potential care. Mater Sci Eng C. 2015;48:651.

33. Ahmadi-Aghkand F, Gholizadeh-Ghaleh AS, Panahi Y, Daraee H, Gorjikhah F, Gholizadeh-Ghaleh AS, Hsanzadeh A, Akbarzadeh A. Recent prospective of nanofiber scaffolds fabrication approaches for skin regeneration. Artif Cells Nanomed Biotechnol. 2016;44:1635.

34. Macmillancrow LA, Crow JP, Kerby JD, Beckman JS, Thompson JA. Nitration and inactivation of manganese superoxide dismutase in chronic rejection of human renal allografts. Proc Natl Acad Sci USA. 1996;93:11853-8

35. Guo S, Dipietro LA. Factors affecting wound healing. Otolaryngol Clin North Am. 1984;17:243.

36. Gainza G, Villullas S, Pedraz JL, Hernandez RM, Igartua M. Advances in drug delivery systems (DDSs) to release growth factors for wound healing and skin regeneration. Nanomedicine. 2015;11:1551-73.

37. Navarro M, Planell JA. Nanotechnology in regenerative medicine. Drug Deliv Syst. 2011;21:623-6.

38. Korrapati PS, Karthikeyan K, Satish A, Krishnaswamy VR, Venugopal JR, Ramakrishna S. Recent advancements in nanotechnological strategies in selection, design and delivery of biomolecules for skin regeneration. Mater Sci Eng C Mater Biol Appl. 2016;67:747-65.

39. Chen J, Cheng D, Li J, Wang Y, Guo JX, Chen ZP, Cai BC, Yang T. Influence of lipid composition on the phase transition temperature of liposomes composed of both DPPC and HSPC. Drug Dev Ind Pharm. 2013;39:197-204

40. Mitragotri S, Burke PA, Langer R. Overcoming the challenges in administering biopharmaceuticals: formulation and delivery strategies. Nat Rev Drug Discov. 2014;13:655-72.

41. Degim Z, Celebi N, Alemdaroglu C, Deveci M, Ozturk S, Ozogul C. Evaluation of chitosan gel containing liposome-loaded epidermal growth factor on burn wound healing. Int Wound J. 2011;8:343-54.

42. Manca ML, Matricardi P, Cencetti C, Peris JE, Melis V, Carbone C, Escribano E, Zaru M, Fadda AM, Manconi M. Combination of argan oil and phospholipids for the development of an effective liposome-like formulation able to improve skin hydration and allantoin dermal delivery. Int J Pharm. 2016;505:204-11.

43. Xu HL, Chen PP, ZhuGe DL, Zhu QY, Jin BH, Shen BX, Xiao J, Zhao YZ. Liposomes with silk fibroin hydrogel core to stabilize bFGF and promote the wound healing of mice with deep second-degree scald. Adv Healthc Mater. 2017;6:1700344.

44. Nunes PS, Rabelo AS, Souza JC, Santana BV, da Silva TM, Serafini MR, Dos Passos Menezes P, Dos Santos Lima B, Cardoso JC, Alves JC, et al. Gelatin-based membrane containing usnic acid-loaded liposome improves dermal burn healing in a porcine model. Int J Pharm. 2016;513:473-82.

45. Li WZ, Hao XL, Zhao N, Han WX, Zhai XF, Zhao Q, Wang YE, Zhou YQ, Cheng YC, Yue YH, et al. Propylene glycol-embodying deformable liposomes as a novel drug delivery carrier for vaginal fibrauretine delivery applications. J Control Release. 2016;226:107-14.

46. Cevc G. Rational design of new product candidates: the next generation of highly deformable bilayer vesicles for noninvasive, targeted therapy. J Control Release. 2012;160:135-46.

47. Choi JU, Lee SW, Pangeni R, Byun Y, Yoon IS, Park JW. Preparation and in vivo evaluation of cationic elastic liposomes comprising highly skin-permeable growth factors combined with hyaluronic acid for enhanced diabetic wound-healing therapy. Acta Biomater. 2017:57:197-215

48. Manconi M, Manca ML, Caddeo C, Valenti D, Cencetti C, Diez-Sales O, Nacher A, Mir-Palomo S, Terencio MC, Demurtas D, et al. Nanodesign of new self-assembling core-shell gellan-transfersomes loading baicalin and in vivo evaluation of repair response in skin. Nanomedicine. 2018;14:569-79.

49. Kianvash N, Bahador A, Pourhajibagher M, Ghafari H, Nikoui V, Rezayat SM, Dehpour AR, Partoazar A. Evaluation of propylene glycol nanoliposomes containing curcumin on burn wound model in rat: biocompatibility, wound healing, and anti-bacterial effects. Drug Deliv Trans| Res. 2017;7:654-63.

50. Magin RL, Niesman MR. Temperature-dependent permeability of large unilamellar liposomes. Chem Phys Lipid. 1984;34:245-56.

51. Gubernator J, Chwastek G, Korycinska M, Stasiuk M, Grynkiewicz G, Lewrick F, Suss R, Kozubek A. The encapsulation of idarubicin within liposomes using the novel EDTA ion gradient method ensures improved drug retention in vitro and in vivo. $J$ Control Release. 2010;146:68-75

52. Sahli A, Cansell M, Tapon-Bretaudière J, Letourneur D, Jozefonvicz J, Fischer AM. The stability of heparin-coated liposomes in plasma and their effect on its coagulation. Colloids Surf B. 1998;10:205-15.

53. Ran R, Middelberg APJ, Zhao CX. Microfluidic synthesis of multifunctional liposomes for tumour targeting. Colloids Surf B Biointerfaces. 2016;148:402-10

54. Huang S, Fu X. Naturally derived materials-based cell and drug delivery systems in skin regeneration. J Control Release. 2010;142:149-59.

55. Yun $\mathrm{YH}$, Goetz DJ, Yellen $\mathrm{P}$, Chen W. Hyaluronan microspheres for sustained gene delivery and site-specific targeting. Biomaterials. 2004;25:147-57.

56. Chu Y, Yu D, Wang P, Xu J, Li D, Ding M. Nanotechnology promotes the full-thickness diabetic wound healing effect of recombinant human epidermal growth factor in diabetic rats. Wound Repair Regen. 2010:18:499-505.

57. Ye M, Kim S, Park K. Issues in long-term protein delivery using biodegradable microparticles. J Control Release. 2010;146:241-60.

58. Gainza G, Aguirre JJ, Pedraz JL, Hernández RM, Igartua M. rhEGFloaded PLGA-Alginate microspheres enhance the healing of fullthickness excisional wounds in diabetised Wistar rats. Eur J Pharm Sci. 2013;50:243-52.

59. Chereddy KK, Her CH, Comune M, Moia C, Lopes A, Porporato PE, Vanacker J, Lam MC, Steinstraesser L, Sonveaux P. PLGA nanoparticles loaded with host defense peptide LL37 promote wound healing. J Control Release. 2014;194:138-47.

60. Dave V, Kushwaha K, Yadav RB, Agrawal U. Hybrid nanoparticles for the topical delivery of norfloxacin for the effective treatment of bacterial infection produced after burn. J Microencapsul. 2017;34:351-65.

61. Sanchez DA, Schairer D, Tuckman-Vernon C, Chouake J, Kutner A, Makdisi J, Friedman JM, Nosanchuk JD, Friedman AJ. Amphotericin B 
releasing nanoparticle topical treatment of Candida spp. in the setting of a burn wound. Nanomedicine. 2014;10:269-77.

62. Yang F, Ferrara N. Genetic engineering of human stem cells for enhanced angiogenesis using biodegradable polymeric nanoparticles. Proc Natl Acad Sci USA. 2010;107:3317-22.

63. Mofazzal Jahromi MA, Sahandi Zangabad P, Moosavi Basri SM, Sahandi Zangabad K, Ghamarypour A, Aref AR, Karimi M, Hamblin MR. Nanomedicine and advanced technologies for burns: preventing infection and facilitating wound healing. Adv Drug Deliv Rev. 2018;123:33-64.

64. Tian J, Wong KK, Ho CM, Lok CN, Yu WY, Che CM, Chiu JF, Tam PK. Topical delivery of silver nanoparticles promotes wound healing. ChemMedChem. 2007:2:129-36.

65. Ali SS, Morsy R, El-Zawawy NA, Fareed MF, Bedaiwy MY. Synthesized zinc peroxide nanoparticles $\left(\mathrm{ZnO}_{2}-\mathrm{NPs}\right)$ : a novel antimicrobial, antielastase, anti-keratinase, and anti-inflammatory approach toward polymicrobial burn wounds. Int J Nanomed. 2017;12:6059-73.

66. Ziv-Polat O, Topaz M, Brosh T, Margel S. Enhancement of incisional wound healing by thrombin conjugated iron oxide nanoparticles. Biomaterials. 2010:31:741-7.

67. Thanusha AV, Dinda AK, Koul V. Evaluation of nano hydrogel composite based on gelatin/HA/CS suffused with Asiatic acid/ZnO and CuO nanoparticles for second degree burns. Mater Sci Eng C Mater Biol Appl. 2018:89:378-86

68. Almeida AJ, Souto E. Solid lipid nanoparticles as a drug delivery system for peptides and proteins. Adv Drug Deliv Rev. 2007;59:478-90.

69. Manjunath K, Venkateswarlu V. Pharmacokinetics, tissue distribution and bioavailability of clozapine solid lipid nanoparticles after intravenous and intraduodenal administration. J Control Release. 2005;107:215-28

70. Silva AC, Amaral MH, González-Mira E, Santos D, Ferreira D. Solid lipid nanoparticles (SLN)-based hydrogels as potential carriers for oral transmucosal delivery of risperidone: preparation and characterization studies. Colloids Surf B. 2012;93:241-8.

71. Souto EB, Müller RH. SLN and NLC for topical delivery of ketoconazole. J Microencapsul. 2005;22:501-10.

72. Gainza G, Pastor M, Aguirre JJ, Villullas S, Pedraz JL, Hernandez RM, Igartua M. A novel strategy for the treatment of chronic wounds based on the topical administration of rhEGF-loaded lipid nanoparticles: in vitro bioactivity and in vivo effectiveness in healing-impaired db/db mice. J Control Release. 2014;185:51-61.

73. Fumakia M, Ho EA. Nanoparticles encapsulated with LL37 and serpin A1 promotes wound healing and synergistically enhances antibacterial activity. Mol Pharm. 2016:13:2318-31.

74. Sanad AB, Abdel-Bar HM. Chitosan-hyaluronic acid composite sponge scaffold enriched with Andrographolide-loaded lipid nanoparticles for enhanced wound healing. Carbohyd Polym. 2017;173:441-50

75. Hromadka M, Collins JB, Reed C, Han L, Kolappa KK, Cairns BA, Andrady T, van Aalst JA. Nanofiber applications for burn care. J Burn Care Res. 2008:29:695.

76. Reddy VJ, Radhakrishnan S, Ravichandran R, Mukherjee S, Balamurugan R, Sundarrajan S, Ramakrishna S. Nanofibrous structured biomimetic strategies for skin tissue regeneration. Wound Repair Regen. 2013:21:1-16

77. Tocco I, Zavan B, Bassetto F, Vindigni V. Nanotechnology-based therapies for skin wound regeneration. J Nanomater. 2012;2012:4.

78. Yang Y, Xia T, Zhi W, Wei L, Weng J, Zhang C, Li X. Promotion of skin regeneration in diabetic rats by electrospun core-sheath fibers loaded with basic fibroblast growth factor. Biomaterials. 2011;32:4243-54.

79. Hu X, Liu S, Zhou G, Huang Y, Xie Z, Jing X. Electrospinning of polymeric nanofibers for drug delivery applications. J Control Release. 2014;185:12-21

80. Adeli-Sardou M, Yaghoobi MM, Torkzadeh-Mahani M, Dodel M. Controlled release of lawsone from polycaprolactone/gelatin electrospun nano fibers for skin tissue regeneration. Int J Biol Macromol. 2019;124:478-91.

81. Shan YH, Peng LH, Liu X, Chen X, Xiong J, Gao JQ. Silk fibroin/gelatin electrospun nanofibrous dressing functionalized with astragaloside IV induces healing and anti-scar effects on burn wound. Int J Pharm. 2015:479:291-301.
82. Geesala R, Bar N, Dhoke NR, Basak P, Das A. Porous polymer scaffold for on-site delivery of stem cells-protects from oxidative stress and potentiates wound tissue repair. Biomaterials. 2016;77:1-13.

83. Zulkifli FH, Hussain FSJ, Zeyohannes SS, Rasad M, Yusuff MM. A facile synthesis method of hydroxyethyl cellulose-silver nanoparticle scaffolds for skin tissue engineering applications. Mater Sci Eng C Mater Biol Appl. 2017;79:151-60

84. Fan X, Chen K, He X, Na L, Huang J, Tang K, Li Y, Fang W. Nano-TiO 2 /Collagen-chitosan porous scaffold for wound repairing. Int J Biol Macromol. 2016;91:15-22.

85. Zhang H, Xia J, Pang X, Zhao M, Wang B, Yang L, Wan H, Wu J, Fu S. Magnetic nanoparticle-loaded electrospun polymeric nanofibers for tissue engineering. Mater Sci Eng C Mater Biol Appl. 2017;73:537-43.

86. Bhattacharya M, Malinen MM, Lauren P, Lou YR, Kuisma SW, Kanninen L, Lille M, Corlu A, Guguen-Guillouzo C, Ikkala O. Nanofibrillar cellulose hydrogel promotes three-dimensional liver cell culture. J Control Release. 2012;164:291-8.

87. Pachuau L. Recent developments in novel drug delivery systems for wound healing. Expert Opin Drug Deliv. 2015;12:1895-909.

88. Anumolu SS, Menjoge AR, Deshmukh M, Gerecke D, Stein S, Laskin J, Sinko PJ. Doxycycline hydrogels with reversible disulfide crosslinks for dermal wound healing of mustard injuries. Biomaterials. 2011;32:1204-17.

89. Hajimiri M, Shahverdi S, Esfandiari MA, Larijani B, Atyabi F, Rajabiani A, Dehpour AR, Amini M, Dinarvand R. Preparation of hydrogel embedded polymer-growth factor conjugated nanoparticles as a diabetic wound dressing. Drug Dev Ind Pharm. 2015;42:1.

90. Manconi M, Manca ML, Caddeo C, Cencetti C, Meo CD, Zoratto N, Nacher A, Fadda AM, Matricardi P. Preparation of gellan-cholesterol nanohydrogels embedding baicalin and evaluation of their wound healing activity. Eur J Pharm Biopharm. 2018;127:244-9.

91. Xi Loh EY, Fauzi MB, Ng MH, Ng PY, Ng SF, Ariffin H, Mohd Amin MCl. Cellular and molecular interaction of human dermal fibroblasts with bacterial nanocellulose composite hydrogel for tissue regeneration. ACS Appl Mater Interfaces. 2018;10:39532-43.

92. Lokhande G, Carrow JK, Thakur T, Xavier JR, Parani M, Bayless KJ, Gaharwar AK. Nanoengineered injectable hydrogels for wound healing application. Acta Biomater. 2018:70:35-47.

93. Fisher DA, Lakshmanan J. Metabolism and effects of epidermal growth factor and related growth factors in mammals. Endocr Rev. 1990;11:418-42.

94. Lindahl P, Johansson BR, Levéen P, Betsholtz C. Pericyte loss and microaneurysm formation in PDGF-B-deficient mice. Science. 1997;277:242-5.

95. Dickson C, Spencerdene B, Dillon C, Fantl V. Tyrosine kinase signalling in breast cancer: fibroblast growth factors and their receptors. Breast Cancer Res. 2000:2:191-6.

96. Gabrilove JL, Jakubowski A, Scher H, Sternberg C, Wong G, Grous J, Yagoda A, Fain K, Moore MA, Clarkson B. Effect of granulocyte colonystimulating factor on neutropenia and associated morbidity due to chemotherapy for transitional-cell carcinoma of the urothelium. N Engl J Med. 1988:318:1414

97. Huang JS, Wang YH, Ling TY, Chuang SS, Johnson FE, Huang SS. Synthetic TGF-beta antagonist accelerates wound healing and reduces scarring. FASEB J. 2002;16:1269-70.

98. Jr BR, Nuccio PA, Wolfe E, Collins B, Tamburro C, Glass W, Martin CM. Relationship of quantitative wound bacterial counts to healing of decubiti: effect of topical gentamicin. Antimicrob Agents Chemother. 1964;10:147.

99. Chopra I, Roberts M. Tetracycline antibiotics: mode of action, applications, molecular biology, and epidemiology of bacterial resistance. Microbiol Mol Biol Rev. 2001;65:232-60.

100. Christersson LA, Norderyd OM, Puchalsky CS. Topical application of tetracycline-HCl in human periodontitis. J Clin Periodontol. 2010;20:88-95.

101. Lipsky BA, Miller B, Schwartz R, Henry DC, Nolan T, Mccabe A, Magner DJ, Talbot GH. Sparfloxacin versus ciprofloxacin for the treatment of community-acquired, complicated skin and skin-structure infections. Clin Ther. 1999;21:675-90.

102. Kemmerly SA, Pankey GA. Oral ciprofloxacin therapy for Bacillus cereus wound infection and bacteremia. Clin Infect Dis. 1993:16:189.

103. Rybak MJ. The pharmacokinetic and pharmacodynamic properties of vancomycin. Clin Infect Dis. 2006;42(Suppl 1):S35-9. 
104. Pohl R, Hunt TK. Penicillin $G$ and wound healing. Arch Surg. 1970;101:610-1.

105. Miller JP, Acar F, Burchiel KJ. Significant reduction in stereotactic and functional neurosurgical hardware infection after local neomycin/polymyxin application. J Neurosurg. 2009:110:247-50.

106. Evans ME, Feola DJ, Rapp RP. Polymyxin B sulfate and colistin: old antibiotics for emerging multiresistant gram-negative bacteria. Ann Pharmacother. 1999;33:960-7.

107. Strock LL, Lee MM, Rutan RL, Desai MH, Robson MC, Herndon DN, Heggers JP. Topical Bactroban (mupirocin): efficacy in treating burn wounds infected with methicillin-resistant staphylococci. J Burn Care Rehabil. 1990;11:454.

108. Wong-Beringer A, Jacobs RA, Guglielmo BJ. Lipid formulations of amphotericin B: clinical efficacy and toxicities. Clin Infect Dis. 1998;27:603-18.

109. Kasten KR, Makley AT, Kagan RJ. Update on the critical care management of severe burns. J Intensive Care Med. 2011;26:223.

110. Church D, Elsayed S, Reid O, Winston B, Lindsay R. Burn wound infections. Clin Microbiol Rev. 2006;19:403.

111. Li Z, Liu M, Wang H, Du S. Increased cutaneous wound healing effect of biodegradable liposomes containing madecassoside: preparation optimization, in vitro dermal permeation, and in vivo bioevaluation. Int J Nanomed. 2016;11:2995-3007.

112. Srinivasulu C, Mughal MR, Eitan O, Soumen D, Amit K, Michael MC, Sudipta S, Mattson MP. Effects of cerium oxide nanoparticles on the growth of keratinocytes, fibroblasts and vascular endothelial cells in cutaneous wound healing. Biomaterials. 2013;34:2194-201.

113. Kim MH, Park H, Nam HC, Park SR, Jung JY, Park WH. Injectable methylcellulose hydrogel containing silver oxide nanoparticles for burn wound healing. Carbohydr Polym. 2018;181:579-86.

114. Perumal S, Ramadass S, Madhan B. Sol-gel processed mupirocin silica microspheres loaded collagen scaffold: a synergistic bio-composite for wound healing. Eur J Pharm Sci. 2014;52:26-33.

115. Karavana SY, Gokce EH, Rencber S, Ozbal S, Pekcetin C, Guneri P, Ertan G. A new approach to the treatment of recurrent aphthous stomatitis with bioadhesive gels containing cyclosporine A solid lipid nanoparticles: in vivo/in vitro examinations. Int J Nanomed. 2012;7:5693-704.

116. Lin J, Li C, Zhao Y, Hu J, Zhang LM. Co-electrospun nanofibrous membranes of collagen and zein for wound healing. ACS Appl Mater Interfaces. 2012:4:1050-7.

117. Rath G, Hussain T, Chauhan G, Garg T, Goyal AK. Development and characterization of cefazolin loaded zinc oxide nanoparticles composite gelatin nanofiber mats for postoperative surgical wounds. Mater Sci Eng C Mater Biol Appl. 2016;58:242-53.

118. Perng CK, Kao CL, Yang YP, Lin HT, Lin WB, Chu YR, Wang HJ, Ma H, Ku $\mathrm{HH}$, Chiou SH. Culturing adult human bone marrow stem cells on gelatin scaffold with pNIPAAm as transplanted grafts for skin regeneration. J Biomed Mater Res Part A. 2010;84A:622-30.

119. Xie Z, Paras CB, Weng H, Punnakitikashem P, Su LC, Vu K, Tang L, Yang J, Nguyen KT. Dual growth factor releasing multi-functional nanofibers for wound healing. Acta Biomater. 2013;9:9351-9.

120. Mao C, Xiang Y, Liu X, Cui Z, Yang X, Kwk Y, Pan H, Wang X, Chu PK, Wu S. Photo-inspired antibacterial activity and wound healing acceleration by hydrogel embedded with Ag/Ag@AgCl/ZnO nanostructures. Acs Nano. 2017;11:9010-21.

121. Moore AN, Silva TLL, Carrejo NC, Marmolejo CAO, Li IC, Hartgerink JD. Nanofibrous peptide hydrogel elicits angiogenesis and neurogenesis without drugs, proteins, or cells. Biomaterials. 2018;161:154.

122. Loo Y, Wong YC, Cai EZ, Ang CH, Raju A, Lakshmanan A, Koh AG, Zhou HJ, Lim TC, Moochhala SM, Hauser CA. Ultrashort peptide nanofibrous hydrogels for the acceleration of healing of burn wounds. Biomaterials. 2014:35:4805-14.

\section{Publisher's Note}

Springer Nature remains neutral with regard to jurisdictional claims in published maps and institutional affiliations.
Ready to submit your research? Choose BMC and benefit from:

- fast, convenient online submission

- thorough peer review by experienced researchers in your field

- rapid publication on acceptance

- support for research data, including large and complex data types

- gold Open Access which fosters wider collaboration and increased citations

- maximum visibility for your research: over $100 \mathrm{M}$ website views per year

At BMC, research is always in progress.

Learn more biomedcentral.com/submissions 